\title{
Decitabine salvage for TP53-mutated, relapsed/refractory acute myeloid leukemia after cytotoxic induction therapy
}

TP53-mutated acute myeloid leukemia (AML) represents a therapeutic challenge due its chemotherapy-refractoriness, and to an uncertain role for hematopoietic cell transplant (HCT) intensification. ${ }^{1}$ We initiated a trial to determine whether decitabine might salvage TP53-mutated AML after failure of cytarabine-based induction. Seventeen patients were enrolled before the trial was closed due to slow accrual. Decitabine was well tolerated in this pretreated population and allowed transition to HCT in seven of 17 patients (41\%). The 1-year overall survival (OS) was 29\% (median 244 days; 95\% confidence interval [Cl]: 116-390). Survival was longer in patients receiving HCT (median 354 days), and two long-term survivors were transplanted in molecular remission. Detection of TP53 clonal response by bone marrow (BM) immunohistochemistry (IHC) or peripheral blood (PB) exome sequencing was associated with improved survival, suggesting the utility of these secondary endpoints in future clinical trials.

This single-arm, open-label, prospective clinical trial (clinicaltrials gov. Identifier: NCT03063203) was approved by the Institutional Review Board at Washington University in St. Louis. The study enrolled 17 patients between October 2017 and September 2020 before closing due to slow accrual during the SARS-CoV-2 pandemic, and to shifting treatment practices towards the use of venetoclax combinations. ${ }^{2}$ Eligible patients had TP53-mutated relapsed/refractory AML following cytarabine-based induction chemotherapy, and at least one of the following: BM blasts $>5 \%$, flow-based measurable residual disease $(M R D)>0.5 \%$, persistent cytogenetic abnormality by fluorescence in situ hybridization (FISH) or karyotyping, or persistent TP53 mutation at $\geq 5 \%$ variant allele frequency (VAF) with exome sequencing. Decitabine was administered at $20 \mathrm{mg} / \mathrm{m}^{2} /$ day on days 1-10 of 28-day cycles and could be reduced to days 1-5 once BM aspirate blasts were $<5 \%$. Granulocyte colony-stimulating factor (G-CSF) use was allowed during the treatment of sepsis and neutropenic fevers, but not to support neutrophil recovery. The primary objective was to determine the 1-year os in patients with TP53-mutated AML compared to historic controls (1-year OS 25\%), ${ }^{1,3,4}$ OS was defined from the time of enrollment to death from any cause. Secondary endpoints included determination of: i) proportion of morphologic responses, as defined by the European LeukemiaNet 2017 (ELN) ${ }^{5}$; ii) time to transplant and the number of patients able to undergo HCT; iii) 2-year eventfree survival after transplant compared to historical controls (18-22\%); ${ }^{6}$ and iv) average number of hospital days during cycles 1-2, as a surrogate of toxicity.
The average time from the initiation of induction chemotherapy to trial enrollment was 42 days (median 25 days), consistent with primary refractory disease, persistent MRD, or rapid relapses after induction chemotherapy (Table 1). Performance status was 0 or 1 in 16 of 17 patients, reflecting a population that had been fit for cytotoxic chemotherapy (Table 1).

Sixteen of the 17 patients had complex cytogenetics and nine of 17 had cytogenetic loss of chromosome 17p (Online Supplementary Table S1). As expected in relapsed/refractory AML and TP53-mutated AML, BM aspirates were commonly hypoplastic, ${ }^{7}$ with a blast count mean of $18 \%$ at trial enrollment and of $37 \%$ at diagnosis. The mean number of hospital days during combined cycles 1 and 2 was 21 (median 14 days; Online Supplementary Table S1), including the inpatient decitabine administration days. Observed grade 3-4 serious adverse events (SAE) reflected typical complications associated with decitabine therapy, including anemia (1), febrile neutropenia (6), heart failure (1), gastrointestinal pain (1), infections (2), abnormal liver function test (LFT) (2), troponin elevation (1), lymphopenia (6), neutropenia (6), thrombocytopenia (4), acidosis (1), hyperglycemia (2), electrolyte imbalance (2), acute kidney injury (2), dyspnea (1), respiratory failure (3), hypertension (1), and hypotension (1).

The median survival was 244 days (95\% Cl: 116-390; Table 1; Figure 1A). Twelve patients died within 1 year of presentation, providing a 29\% 1-year OS. An interim analysis noted $27 \%$ predictive power to reject the null hypothesis if the study were to include 60 patients $^{8}$ (calculated using PASS v.15.0.5). All non-transplanted patients eventually relapsed and died of disease progression. Seven patients underwent HCT, at a mean of 106 days (median 117 days, Online Supplementary Table S1). Three patients died in remission from complications of transplantation, two died after relapse/progression (Online Supplementary Table S1). Two patients remain alive at 26 and 18 months; both were transplanted in molecular remission. Overall, HCT was associated with longer survivals (median 354 days; Figure $1 \mathrm{~B})$. No patient achieved a complete morphologic response by ELN criteria (Table 1). Robust neutrophil recovery was not noted, though G-CSF was not used to treat asymptomatic neutropenia. Five patients displayed platelet counts normalization (Figure 1C).

$\mathrm{BM}$ and $\mathrm{PB}$ samples were collected at enrollment (day 0 ) and at the end of cycles 1, 2, and 3. For 16 patients, samples at AML diagnosis (pre-induction) were also available for correlative studies. TP53 IHC (antibody clone D07) was performed on $4 \mu \mathrm{m}$ BM sections using the Benchmark 
XT automated stainer (Ventana Medical Systems, Tucson, AZ, USA). Quantitative scoring was performed on nuclear staining in 500 hematopoietic cells. Based on published cutoffs, ${ }^{9}$ IHC response was defined as a reduction of
TP53+ cells to $<10 \%$ of total cells on a core biopsy sample. Many TP53 missense mutations are associated with IHCdetectable TP53, via protein stabilization. ${ }^{10,11}$ TP53 IHC showed staining in all cases with TP53 missense muta-

Table 1. Clinical characteristics and treatment responses.

\begin{tabular}{|c|c|c|c|}
\hline Variable & Level & $N=17$ & $\%$ \\
\hline \multirow[t]{2}{*}{ Sex } & $\mathrm{F}$ & 6 & 35.3 \\
\hline & $M$ & 11 & 64.7 \\
\hline Age in years (range) & Median & $61(43-74)$ & \\
\hline \multirow{3}{*}{ ECOG Performance Status } & 0 & 4 & 23.5 \\
\hline & 1 & 12 & 70.5 \\
\hline & 2 & 1 & 6 \\
\hline $\begin{array}{l}\text { Days from induction to } \\
\text { enrollment (range) }\end{array}$ & Median & $25(16-176)$ & \\
\hline \multirow[t]{3}{*}{ Induction chemotherapy } & $7+3$ & 6 & \\
\hline & Vyxeos (CPX-351) & 10 & \\
\hline & FLAG & 1 & \\
\hline \multirow[t]{2}{*}{ 1-year OS } & died before 1 year & 12 & $71 \%$ \\
\hline & survived > 1 year & 5 & $29 \%$ \\
\hline \multirow{2}{*}{$\begin{array}{l}\text { Transplant (day of treatment) } \\
(\mathrm{n}=7)\end{array}$} & Median & 117 & \\
\hline & Mean & 106 & \\
\hline \multirow{2}{*}{ Survival post transplant (days) } & Median & 254 & \\
\hline & Mean & 236 & \\
\hline \multirow[t]{5}{*}{ ELN responses } & CRi & 5 & $29.4 \%$ \\
\hline & mLFS & 1 & $5.9 \%$ \\
\hline & SD & 6 & $35.3 \%$ \\
\hline & PD & 2 & $11.8 \%$ \\
\hline & NA & 3 & $17.6 \%$ \\
\hline
\end{tabular}

7+3: 7 days cytarabine, 3 days anthracycline. FLAG: fludarabine, cytarabine, idarubicin and granulocyte colony stimulating factor; CRi: complete remission with incomplete count recovery; mLFS: morphologic leukemia free state; SD: stable disease; PD: progressive disease; NA: not possible to evaluate.

\section{A All patients}

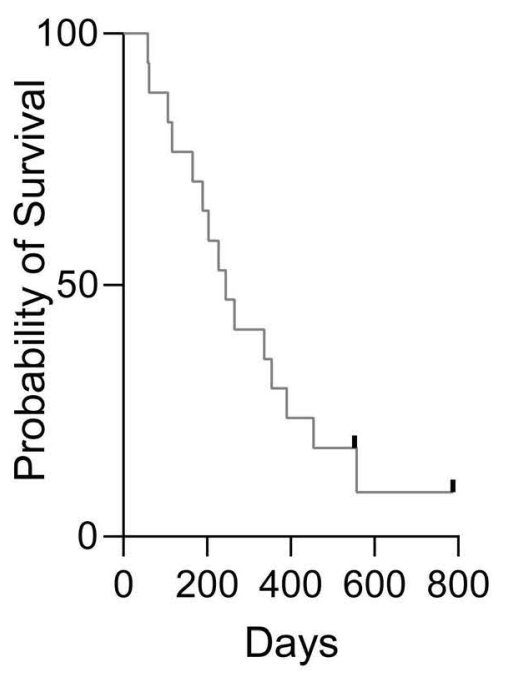

B Transplanted patients

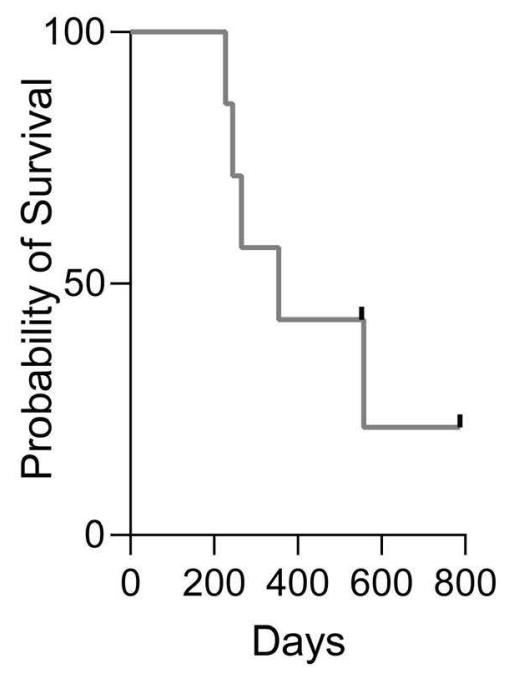

C Platelets

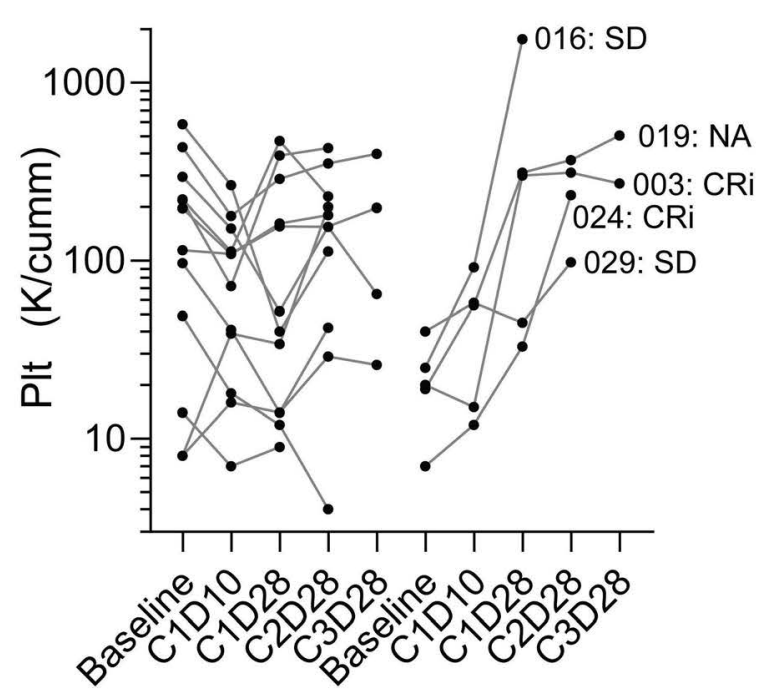

Stable plt vs. plt recovery

Figure 1. Summary of clinical responses. (A) Kaplan-Meier curve describing the overall survival of the 17 patients enrolled in the trial (median survival 244 days, 95\% confidence interval: 116-390 days). (B) Kaplan-Meier curve describing the overall survival of the seven transplanted patients (median survival 354 days). (C) Line plots showing the platelet (Plt) count trends at different time points for the 17 the patients enrolled in the study. On the right side of the plot are separately displayed five patients (003, 016, 019, 024 and 029) with platelet recovery. SD: stable disease; NA: not possible to evaluate; CRi: complete remission with incomplete count recovery. 
A IHC responders

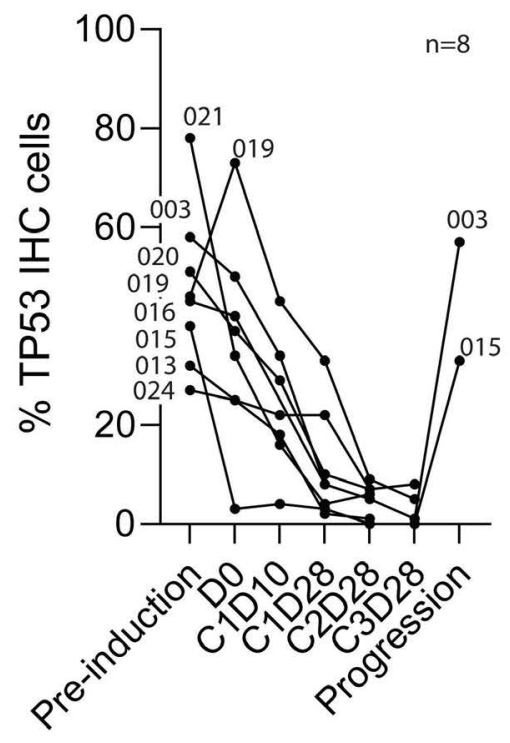

C VAF responders

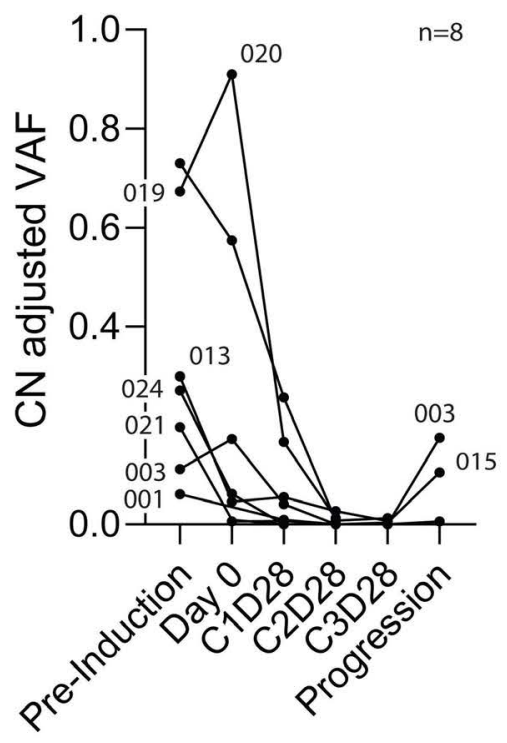

E

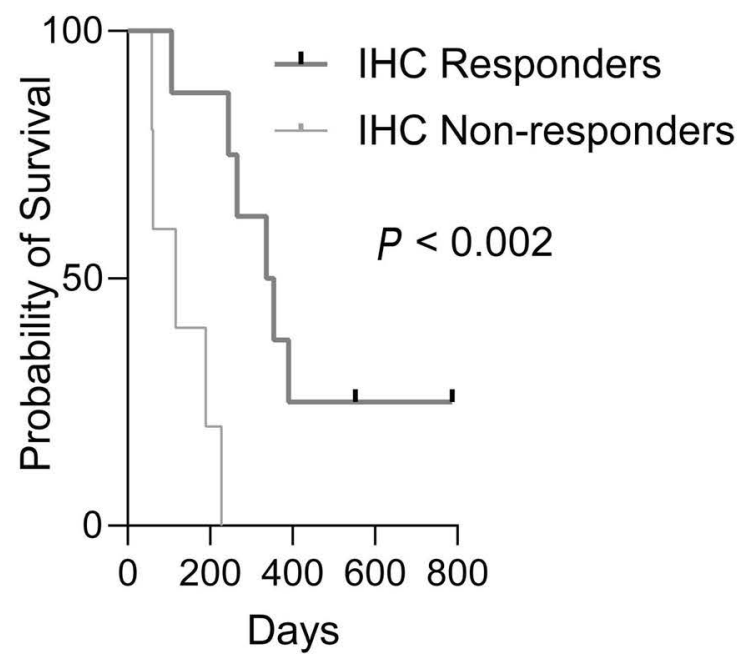

B IHC non-responders

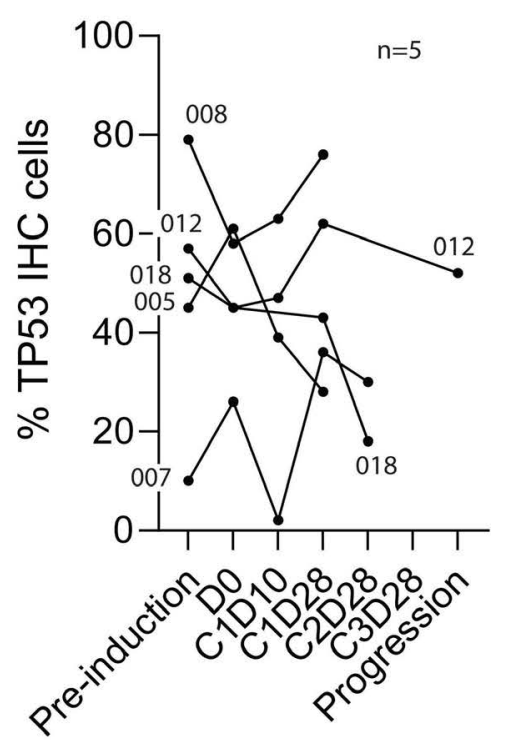

D VAF non-responders

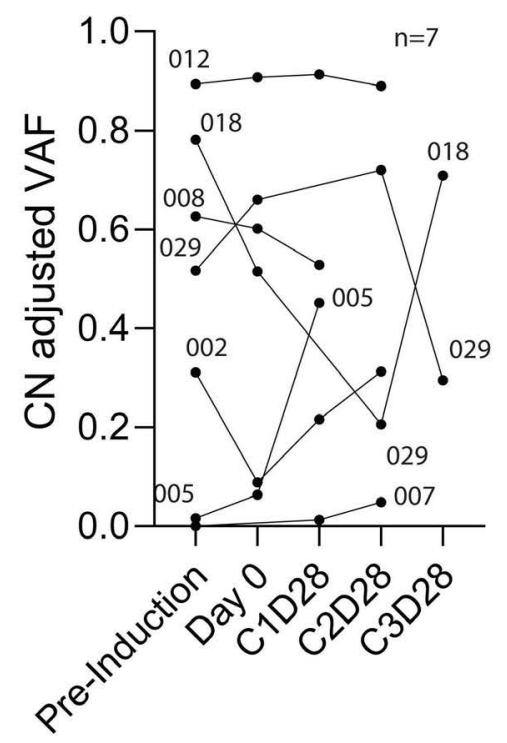

Figure 2. Molecular responses trends and their association with overall survival. ( $A$ and $B$ ) Line plots showing the \% TP53-positive bone marrow cells by immunohistochemistry (IHC) at different time points of response assessment for: responder cases (IHC TP53 $<10 \%$ reduction during therapy) vs. non-responder cases (IHC TP53 >10\% during therapy). (C and D) Line plots of TP53 variant allele frequency (VAF) during therapy for responder cases ((E) reduction of copy number (CN) adjusted TP53 VAF below 0.05\%) vs. nonresponder cases ((F) VAF stable or progressing over time). (E) Overall survival curves of the 17 cases stratified by responses assessed with IHC (median OS 345 days vs. 116 days, $P<0.002$ ). (F) Overall survival stratified by responses assessed with exome sequencing (median OS 390 days vs. 165 days; $P<0.001)$.

\section{F TP53 Mutation Response}

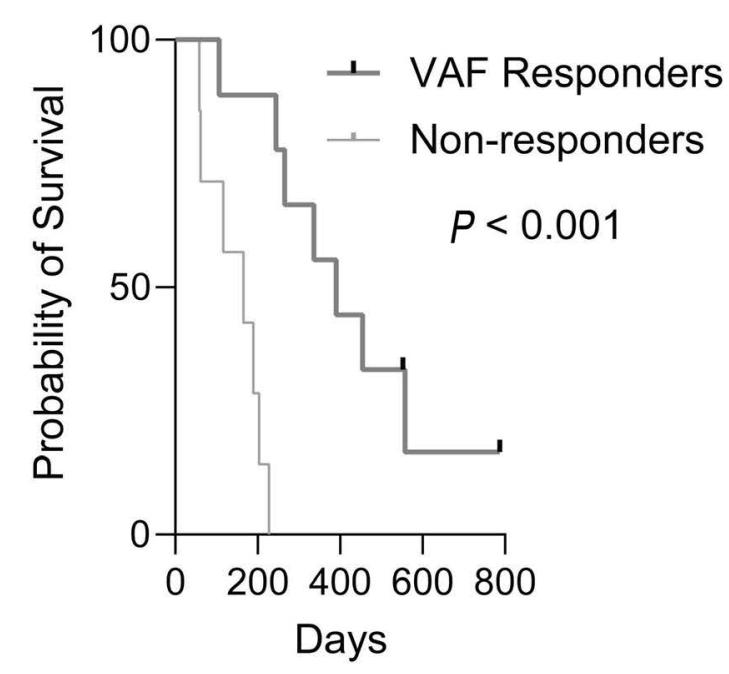

tions; however, three of four cases with nonsense mutations did not have detectable TP53 by IHC (R213*, Y107*, F54Sfs*69; Online Supplementary Figure S1A to C). Unexpectedly, the C-terminal nonsense mutation (R342*) led to elevated TP53 protein at three separate time points (Online Supplementary Figure S1D). TP53 IHC protein levels correlated between pre-induction and day $0\left(R^{2}=0.56\right)$. Only two cases (WUDAC015 and WUDAC021) were associ- ated with a significant reduction of TP53 IHC staining after cytotoxic induction therapy, confirming the limited efficacy of standard induction in this cohort (Online Supplementary Figure S1E and F). Serial assessment of response by IHC noted eight patients with responses (Figure 2A), and five patients without responses (Figure 2B). Four patients could not be evaluated: three had nonsense variants (WUDAC001, WUDAC002, and WUDAC029; Online 
Supplementary Figures S1A to C), and one (WUDAC014) had del17p, all resulting in absent IHC TP53 staining.

Exome sequencing was performed in parallel using $\mathrm{PB}$ samples to circumvent sampling variation due to hemodilute collections in this hypocellular disease (Online Supplementary Table S1). Exome capture utilized an IDT exome reagent, and was resolved on an Illumina NovaSeq S4 300XP to a median depth of $200 x$ for pre-induction and day 0 samples, and 148x (range, 76 - 200x) for other time points. This provided $>100 x$ coverage for $>90 \%$ of targets in 61 of 67 samples. Molecular response was defined as a reduction in the copy number adjusted TP53 to a VAF of $<0.05^{12}$. The computational pipeline is available at https://github.com/genome/analysisworkflows/blob/968d7d80c3cec865c7fa58b4dc24561a4d bfd9ad/definitions/pipelines/somatic_exome.cwl. Mutation burden assessment at day 0 and at pre-induction showed correlation $\left(R^{2}=0.8\right)$. Eight patients achieved a molecular response, and seven patients displayed persistence of TP53 mutations after therapy (Figure $2 \mathrm{C}$ and D). For one patient (WUDAC016), no follow-up PB samples were available (Online Supplementary Figure S1G).

The absolute TP53 tumor burden quantified by BM IHC, either at pre-induction or at day 0 , did not consistently correlate with $\mathrm{PB}$ exome results $\left(\mathrm{R}^{2}=0.15\right.$ and 0.27 , respectively). However, qualitative response trends were concordant in 11 of 16 evaluable patients (Online Supplementary Figures S1D to $F$ and S1H to O). One case (WUDAC005; Online Supplementary Figure S1P) showed stable disease by TP53 IHC, but progressive disease by PB exome sequencing, suggesting peripheralization of AML cells during therapy.

Exome analyses revealed that the global molecular and clinical response was dictated by the TP53 clonal response trend (Online Supplementary Figure S2). Discordance between the TP53 clone and an alternate clone was only observed in WUDAC001, who progressed with a different clone during TP53 clonal response.

Survival outcomes were longer in patients with molecular responses identified by TP53 IHC (median OS 345 days vs. 116 days, $P<0.002$; Figure $2 \mathrm{E}$ ) or by exome sequencing (median OS 390 days vs. 165 days, $P<0.001$; Figure 2F). These results are consistent with data from other studies, ${ }^{13}$ and suggest that IHC and exome sequencing could be useful adjunctive strategies to quantify responses in future clinical trials. However, each approach has limitations: IHC is applicable only to cases with mutations that stabilize TP53 protein (typically missense variants) and lacks specificity below tumor burden of $10 \%$, due to background staining that occurs in a small number of non-malignant cells. Sequencing of PB samples qualitatively reflected the measurement of TP53 levels in the BM in this study, however this approach is affected by the proportion of circulating malignant cells.
TP53-mutated AML has dismal outcomes and is commonly associated with chemotherapy resistance. Although we found that decitabine is tolerated after intensive chemotherapy, and that molecular responses are achievable in a subset of relapsed/refractory TP53 patients, long-term survival remained poor. These results are consistent with prior studies reporting lower responses to decitabine in relapsed/refractory disease versus untreated cases. ${ }^{14,15}$ Therefore, novel therapies, upfront combination and consolidation strategies should be considered. The hypoplastic BM in many patients makes accurate response determination challenging, due to hemodilute aspirate collections. The integration of molecular endpoints into clinical trials may improve response quantification, and increase the ability to identify significant differences between treatment arms.

\section{Authors}

Francesca Ferraro, ${ }^{1}$ Agata Gruszczynska, ${ }^{1}$ Marianna B. Ruzinova, ${ }^{2}$ Christopher A. Miller, ${ }^{1}$ Mary Elizabeth Percival,, ${ }^{3,4}$ Geoffrey L. Uy, Iskra Pusic, ${ }^{1}$ Meagan A. Jacoby, ${ }^{1}$ Mathew J. Christopher, ${ }^{1}$ Miriam Y. Kim, ${ }^{1}$ Peter Westervelt, ${ }^{1}$ Amanda F. Cashen, ${ }^{1}$ Mark A. Schroeder, John F. DiPersio, ${ }^{1}$ Camille N. Abboud, ${ }^{1}$ Lukas D. Wartman, ${ }^{1}$ Feng Gao, ${ }^{5}$ Daniel C. Link, Timothy J. Ley ${ }^{1}$ and John S. Welch ${ }^{1}$

${ }^{1}$ Department of Medicine, Division of Oncology, Washington University School of Medicine, St. Louis, MO; ${ }^{2}$ Department of Pathology and Immunology, Washington University School of Medicine, St. Louis, MO; ${ }^{3}$ Department of Internal Medicine, Division of Hematology, University of Washington School of Medicine, Seattle, WA; ${ }^{4}$ Clinical Research Division, Fred Hutchinson Cancer Research Center, Seattle, WA and ${ }^{5}$ Department of Surgery, Division of Public Health Sciences, Washington University School of Medicine, St. Louis, MO, USA.

Correspondence:

John S. Welch - jwelch@dom.wustl.edu

https://doi.org/10.3324/haematol.2021.280153

Received: October 7, 2021.

Accepted: February 21, 2022.

Prepublished: March 3, 2022.

\section{Disclosures}

No conflicts of interest to disclose.

\section{Contributions}

FF and JSW designed the trial, wrote the manuscript and oversaw the study; MEP, GLU, IP, MAJ, MYK, PW, AFC, MAS, JFD, CNA and LDW contributed to clinical enrollment and data analysis; $A G, M B R$, CAM, DCL, and TJL contributed to molecular analysis; FG contributed to statistical analysis. All authors had access to primary data, reviewed and approved the manuscript. 


\section{Acknowledgments}

We thank Megan Haney and Jeff King for assistance in patient enrollment, sample collection, and data processing; Sharon Heath, Nicole Helton, and the Tissue Procurement Core for assistance in sample collection and processing; the McDonnell Genome Institute at Washington University in St. Louis for support in sequencing; and Anh Vu for help with mutation manual review.

\section{Funding}

The study was supported by grants from the Specialized Program of
Research Excellence in AML of the National Cancer Institute (P50 CA171963 to DCL), the Genomics of AML Program Project (P01 CA101937, to TJL), a K12 Program grant (CA167540, to FF) and by Janssen Pharmaceuticals.

\section{Data sharing statement}

Data sharing is subject to Institutional policies governing clinical research data. Data can be made available upon request to the corresponding author stating dbGaP study ID phs000159.

\section{References}

1. Rucker FG, Schlenk RF, Bullinger L, et al. TP53 alterations in acute myeloid leukemia with complex karyotype correlate with specific copy number alterations, monosomal karyotype, and dismal outcome. Blood. 2012;119(9):2114-2121.

2. Maiti A, Qiao W, Sasaki K, et al. Venetoclax with decitabine vs intensive chemotherapy in acute myeloid leukemia: a propensity score matched analysis stratified by risk of treatment-related mortality. Am J Hematol. 2021;96(3):282-291.

3. Papaemmanuil E, Gerstung M, Bullinger L, et al. Genomic classification and prognosis in acute myeloid leukemia. $N$ Engl $J$ Med. 2016;374(23):2209-2221.

4. Stengel A, Kern W, Haferlach T, et al. The impact of TP53 mutations and TP53 deletions on survival varies between AML, ALL, MDS and CLL: an analysis of 3307 cases. Leukemia. 2017;31(3):705-711.

5. Dohner H, Estey E, Grimwade D, et al. Diagnosis and management of AML in adults: 2017 ELN recommendations from an international expert panel. Blood. 2017;129(4):424-447.

6. Middeke JM, Beelen D, Stadler M, et al. Outcome of high-risk acute myeloid leukemia after allogeneic hematopoietic cell transplantation: negative impact of abnl(17p) and $-5 / 5 q$. Blood. 2012;120(12):2521-2528.

7. Welch JS. Patterns of mutations in TP53 mutated AML. Best Pract Res Clin Haematol 2018;31(4):379-383.

8. Jennison CaT, Bruce W. Group sequential methods with applications to clinical trials. Chapman \& Hall; 2000.
9. Sallman DA, DeZern AE, Garcia-Manero G, et al. Eprenetapopt (APR-246) and azacitidine in TP53-mutant myelodysplastic syndromes. J Clin Oncol. 2021;39(14):1584-1594.

10. Ruzinova MB, Lee YS, Duncavage EJ, Welch JS. TP53 immunohistochemistry correlates TP53 mutation status and clearance in decitabine-treated patients with myeloid malignancies. Haematologica. 2019;104(8):e345-e348.

11. McGraw KL, Nguyen J, Komrokji RS, et al. Immunohistochemical pattern of p53 is a measure of TP53 mutation burden and adverse clinical outcome in myelodysplastic syndromes and secondary acute myeloid leukemia. Haematologica. 2016;101(8):e320-e323.

12. Klco JM, Miller CA, Griffith M, et al. Association between mutation clearance after induction therapy and outcomes in acute myeloid leukemia. JAMA. 2015;314(8):811-822.

13. Hunter AM, Komrokji RS, Yun S, et al. Baseline and serial molecular profiling predicts outcomes with hypomethylating agents in myelodysplastic syndromes. Blood Adv. 2021;5(4):1017-1028.

14. DiNardo CD, Maiti A, Rausch CR, et al. 10-day decitabine with venetoclax for newly diagnosed intensive chemotherapy ineligible, and relapsed or refractory acute myeloid leukaemia: a singlecentre, phase 2 trial. Lancet Haematol. 2020;7(10):e724-e736.

15. Welch JS, Petti AA, Miller CA, et al. TP53 and decitabine in acute myeloid leukemia and myelodysplastic syndromes. N Engl J Med. 2016;375(21):2023-2036. 